\title{
Venus nitric oxide nightglow mapping from SPICAV nadir observations
}

\author{
A. Stiepen ${ }^{\text {a,* }}$, J.-C. Gérard ${ }^{\text {a }}$, M. Dumont ${ }^{\text {a }}$, C. Cox ${ }^{\text {a }}$, J.-L. Bertaux ${ }^{\text {b }}$ \\ ${ }^{a}$ Laboratoire de Physique Atmosphérique et Planétaire Université de Liège, Allée du 6 Août, 17, B5c, 4000 Liège, Belgium \\ ${ }^{\mathrm{b}}$ Laboratoire Atmosphères, Milieux, Observations Spatiales (LATMOS), Université de Versailles Saint-Quentin, Boulevard d'Alembert, 78280 Guyancourt, France
}

\section{A R T I C L E I N F O}

\section{Article history:}

Received 6 March 2013

Revised 6 May 2013

Accepted 17 May 2013

Available online 2 June 2013

\section{Keywords:}

Ultraviolet observations

Venus

Venus, atmosphere

Atmospheres, Dynamics

Atmospheres, Structure

\begin{abstract}
A B S T R A C T
Nitric oxide $\delta(190-240 \mathrm{~nm})$ and $\gamma(255-270 \mathrm{~nm})$ bands have been observed on the Venus nightside with Venus Express SPICAV instrument operated in the nadir mode. These ultraviolet emissions arise from the de-excitation of NO molecules created by radiative recombination of $\mathrm{O}\left({ }^{3} \mathrm{P}\right)$ and $\mathrm{N}\left({ }^{4} \mathrm{~S}\right)$ atoms. These atoms are produced on the dayside of the planet through photodissociation of $\mathrm{CO}_{2}$ and $\mathrm{N}_{2}$ molecules and are transported to the nightside by the global subsolar to antisolar circulation. We analyze a wide dataset of nadir observations obtained since 2006 to determine the statistical distribution of the NO nightglow and its variability. Individual observations show a great deal of variability and may exhibit multiple maxima along latitudinal cuts. We construct and compare a global NO map with the results obtained during the Pioneer-Venus mission and with the recently observed $\mathrm{O}_{2}\left(\mathrm{a}^{1} \Delta_{\mathrm{g}}\right)$ nightglow distribution. The NO airglow distribution shows a statistical bright region extending from 01:00 to 03:30 local time and $25^{\circ} \mathrm{S}$ to $10^{\circ} \mathrm{N}$, very similar to the Pioneer results obtained 35 years earlier during maximum solar activity conditions. The shift from the antisolar point and the difference with the $\mathrm{O}_{2}$ airglow indicate that superrotating zonal winds are statistically weak near $97 \mathrm{~km}$, but play an important role near $115 \mathrm{~km}$. We compare these results with other evidence for superrotation in the thermosphere and point out possible sources of momentum transfer.
\end{abstract}

(c) 2013 Elsevier Inc. All rights reserved.

\section{Introduction}

Observations of planetary airglow emissions are a useful tool to remotely probe the characteristics of upper atmospheres. Indeed, the study of airglow morphology, its temporal or geographical variations and its brightness provide valuable information concerning the composition, temperature and dynamics of an atmosphere. In the case of Venus, the presence of the $\gamma(225-270 \mathrm{~nm})$ and $\delta$ $(190-240 \mathrm{~nm})$ bands of nitric oxide in the nightglow was detected and identified by Feldman et al. (1979) using the ultraviolet spectrograph on board the International Ultraviolet Explorer (IUE) satellite. It was also observed by Stewart and Barth (1979) with the ultraviolet spectrometer on board the Pioneer Venus Orbiter (PVOUVS). This emission is produced by radiative recombination through inverse predissociation of nitrogen $\left({ }^{4} \mathrm{~S}\right)$ and oxygen $\left({ }^{3} \mathrm{P}\right)$ ground-state atoms. These atoms recombine producing NO molecules in the $\left(C^{2} \Pi\right)$ state that can relax following the scheme:

$\mathrm{O}\left({ }^{3} \mathrm{P}\right)+\mathrm{N}\left({ }^{4} \mathrm{~S}\right) \rightarrow \mathrm{NO}\left(\mathrm{C}^{2} \Pi\right)$

$\mathrm{NO}\left(\mathrm{C}^{2} \Pi\right) \rightarrow \mathrm{NO}\left(\mathrm{X}^{2} \Pi\right)+\delta$ bands

$\mathrm{NO}\left(\mathrm{C}^{2} \Pi\right) \rightarrow \mathrm{NO}\left(\mathrm{A}^{2} \Sigma, \mathrm{v}^{\prime}=0\right)+1.22 \mu \mathrm{m}$

$\mathrm{NO}\left(\mathrm{A}^{2} \Sigma, \mathrm{v}^{\prime}=0\right) \rightarrow \mathrm{NO}\left(\mathrm{X}^{2} \Pi\right)+\gamma$ bands

\footnotetext{
* Corresponding author.

E-mail address: arnaud.stiepen@ulg.ac.be (A. Stiepen).
}

The widely accepted global picture is that the $\mathrm{N}$ and $\mathrm{O}$ atoms are mainly created by dissociation of $\mathrm{N}_{2}, \mathrm{CO}_{2}$ and, to a minor extent, $\mathrm{CO}$ molecules on the dayside of Venus by extreme ultraviolet (EUV) photons and photoelectrons. $\mathrm{N}\left({ }^{4} \mathrm{~S}\right)$ and $\mathrm{O}\left({ }^{3} \mathrm{P}\right)$ atoms are then carried to the nightside by the overall global wind circulation. Downward transport takes place on the nightside, followed by radiative recombination providing excited $\mathrm{NO}\left(\mathrm{C}^{2} \Pi\right)$ molecules in the lower thermosphere.

Stewart et al. (1980) analyzed images of the Venus nightside $\delta(0,1)$ band at $198 \mathrm{~nm}$ obtained every $24 \mathrm{~h}$ with PV-OUVS during a solar maximum period and showed that the emission is highly variable in brightness and morphology over consecutive observations. The location of bright spots in successive global images ranged from 2130 to $0300 \mathrm{LT}$ and $39^{\circ} \mathrm{S}$ to $60^{\circ} \mathrm{N}$, as was confirmed by Bougher et al. (1990). They constructed a global map of the NO UV nightglow showing that the emission is statistically concentrated in a bright spot located near $0200 \mathrm{LT}, 10-20^{\circ}$ south of the equator. After revisions by Bougher et al. (1990), the estimated vertical emission rate of this bright spot emission was $\sim 1.9$ kiloRayleighs $(\mathrm{kR})$ for the $\delta(0,1)$ band, whereas the average hemispheric nightside intensity was $0.45 \mathrm{kR}$ for this emission band (see Table 1 ). These observations confirmed the general picture of the Venusian $\mathrm{N}$ and $\mathrm{O}$ global circulation. This concept was numerically validated by a three-dimensional model using the Venus Thermospheric General Circulation Model (VTGCM) (Bougher et al., 1990). The statistical location of the bright spot was well predicted by this 
three-dimensional model and implied retrograde zonal winds of $\sim 50-75 \mathrm{~ms}^{-1}$ in the $115-150 \mathrm{~km}$ altitude region. The observed shift toward dawn of the statistical location of the airglow maximum was also well reproduced by the VTGCM and interpreted as a persistence of the atmospheric zonal superrotation into the thermosphere. The presence of a helium bulge (Niemann et al., 1980) near the morning terminator appeared consistent with the persistence of a dawnward zonal component into the thermosphere. Niemann et al. (1980) Figure 13 sketched a possible flow pattern in the nightside thermosphere. They proposed that the Coriolis force may divert the flow vectors of the superrotation to a stagnation point at low latitudes close to the morning terminator. Brecht et al. (2011) updated the original VTGCM and decreased the altitude level of the lower boundary to model both the NO and the $\mathrm{O}_{2}\left(\mathrm{a}^{1} \Delta\right)$ emissions. They summarized the existence of two different dominating circulation patterns in the Venus upper atmosphere (Dickinson and Ridley, 1977; Schubert et al., 1980, 2007; Bougher et al., 1997, 2006; Lellouch et al., 1997). For an altitude range from the surface to $\sim 70 \mathrm{~km}$, the retrograde superrotating zonal (RSZ) flow dominates. This wind flows in the direction of the planetary spin and is faster than Venus' rotation. Above $\sim 120 \mathrm{~km}$, the SSAS flow pattern is dominant. In the intermediate altitude region, these two flows are thought to be superimposed with relative contributions varying in time and space.

Limb observations of the NO $\gamma$ and $\delta$ emission bands performed by SPICAV have been reported and analyzed by Gérard et al. (2008) and Stiepen et al. (2012). The mean altitude of the emission layer was found at $115 \mathrm{~km}$, with large variations, in agreement with Gérard et al. (1981) who found a peak altitude of $115 \pm 2 \mathrm{~km}$ from the analysis of PV-OUVS limb scans. The limb brightness depends on latitude and local time and is highly variable with a mean value of $60 \mathrm{kR}$. Stiepen et al. (2012) used SPICAV grazing limb observations to determine the volume emission rate (VER - in photons $\mathrm{cm}^{-3} \mathrm{~s}^{-1}$ ) of NO through the Abel inversion technique. They analyzed the distribution of the deconvolved peak intensity and altitude of the NO UV nightglow and VER, as well as its dependence on latitude, local time, etc. They found that the highest VER values are observed around 0200 LT. Unfortunately, as a consequence of orbital characteristics, limb scans are limited to the northern hemisphere. The highest VER values are located close to the equator in the northern hemisphere. They defined an angle from the statistical brightest spot (ABS) centered on 0200 local time and $10-20^{\circ} \mathrm{S}$ latitude and found a decreasing trend of the VER intensities with increasing ABS values, thus confirming the presence of the statistical brightest spot previously observed by Stewart et al. (1980). Altitude and brightness information have also been retrieved by Royer et al. (2010) by analyzing the additional atmospheric signal observed during slitless stellar occultation measurements made with the SPICAV instrument. They also used a forward model to derive a peak altitude of $113.5 \pm 6 \mathrm{~km}$, a topside scale height of $3.4 \pm 1 \mathrm{~km}$ and a brightness ranging from 19 to $540 \mathrm{kR}$ for the vertical distribution of the NO UV emissions, in full agreement with the direct spectral observations.

Soret et al. (2010) constructed a global map of the $\mathrm{O}_{2}\left(\mathrm{a}^{1} \Delta\right)$ emission around $96.5 \mathrm{~km}$ altitude by assembling nadir images taken by the VIRTIS-M-IR instrument (Drossart et al., 2007; Piccioni et al., 2009) on board Venus Express. They also extracted emission profiles of the $1.27 \mu \mathrm{m}$ emission from limb images obtained by VIRTIS. They derived the equivalent vertical intensity from these profiles by vertical integration of the local emission rate derived from the Abel inversion of the limb profiles. They combined nadir profiles in the southern hemisphere with limb observations in the northern hemisphere to produce a statistical global map of the $\mathrm{O}_{2}\left(\mathrm{a}^{1} \Delta\right)$ emission. They found that the region of enhanced emission is located around the antisolar point (Soret et al., 2010, Fig. 1), with a maximum brightness of $1.6 \mathrm{MR}$ and dropping with decreasing solar zenith angle. The mean hemispheric brightness at the nadir of the $\mathrm{O}_{2}\left(\mathrm{a}^{1} \Delta\right)$ nightglow was found to be $0.5 \mathrm{MR}$. The position of the brightest region was previously observed by ground observations (Crisp et al., 1996; Ohtsuki et al., 2005, 2008). This result is counter-intuitive and contradicts the 3-D models (e.g. Bougher et al., 1990) results predicting that superrotation of the atmosphere vanishes in the lower thermosphere. It indicates that the superrotating zonal flow is statistically quite weak near $96 \mathrm{~km}$, but picks up strength again at higher altitude.

In this study, we analyze SPICAV ultraviolet observations collected in the nadir direction in order to construct a global map of the NO emission and analyze its spatial distribution. Our main purpose is to compare the morphology of the nightside NO airglow observed with Venus Express during the low to medium solar activity period 2006-2011 with that observed with PV-OUVS over 30 years ago at high activity. In particular the question arises whether the statistical location of the bright spot was still shifted dawnward from the antisolar point. We first describe the geometry of the observations, signal processing and construction of the airglow map. We then compare these results with those of Pioneer Venus and the recent Venus Express observations of the $\mathrm{O}_{2}$ Infrared Atmospheric band at $1.27 \mu \mathrm{m}$. Finally, we draw some conclusions about the variability of the atmospheric dynamics in the mesosphere-thermosphere transition region.

\section{Nadir observations}

The Venus Express (VEx) spacecraft (Svedhem et al., 2007)) of the European Space Agency has been orbiting Venus along a quasi-polar eccentric trajectory with a period of $24 \mathrm{~h}$ since April 2006. The apocenter is located at $66,000 \mathrm{~km}$, while the altitude of the pericenter varies between $250 \mathrm{~km}$ and $185 \mathrm{~km}$. The orbit is fixed in the inertial space and therefore precesses at the rate of $1.6^{\circ}$ per day. As a consequence of the precession of the orbital plane with regard to the direction of the Sun, a very large variety of configurations exists on both the day and night sides of the planet. The Spectroscopy for Investigation of Characteristics of the Atmosphere of Venus (SPICAV) ultraviolet spectrometer has been extensively described by Bertaux et al. (2007). SPICAV-UV covers a spectral range extending from $118 \mathrm{~nm}$ to $320 \mathrm{~nm}$, entirely including the NO $\gamma(225-270 \mathrm{~nm})$ and $\delta(190-240 \mathrm{~nm}))$ bands. The detector is a $288 \times 407$ pixel CCD and the angular field of view of one pixel is $0.7 \times 0.7$ arcmin. For reasons of telemetry limitations and because of the time needed to read all the lines of the CCD, five adjacent zones of the CCD detector are usually read out. Several observation modes may be selected, namely nadir observations, star pointing for stellar occultations by the Venus atmosphere, fixed point tracking and limb observations, all described by Titov et al. (2006). These data are available from ESA's planetary science archives (http://www.rssd.esa.int/index.php_project=PSA\&page= home).

In the nadir observation mode, the width of each spatial bin of SPICAV-UV is 32 pixel lines, corresponding to a field of view of $1.7^{\circ}$. These lines are seen through the large slit $(500 \mu \mathrm{m})$, providing a spectral resolution of $\sim 15 \mathrm{~nm}$. The planetary area intercepted by the field of view depends on the location of the spacecraft on its orbits and it must be calculated for each individual spectrum. The non-uniform dark current and offset values are carefully subtracted from each individual spectrum, using similar observations performed with a null amplification of the UV image intensifier (see Appendix A). The absolute calibration obtained by observing well-known hot stars spectra is then applied to obtain emission rates of the total gamma and delta NO emission expressed in kiloRayleighs (kR). Other intermediate steps are necessary before building up a global statistical airglow map. 


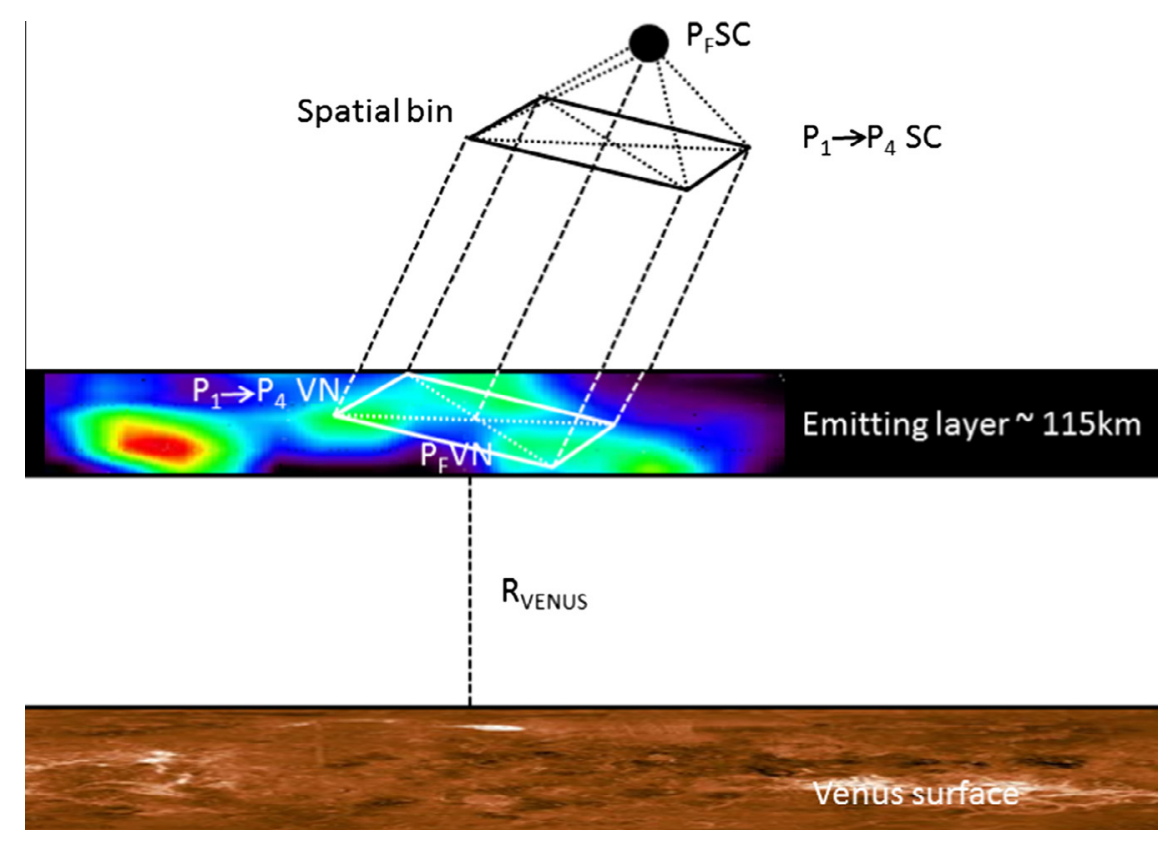

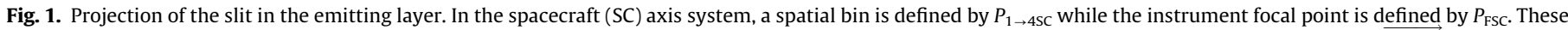

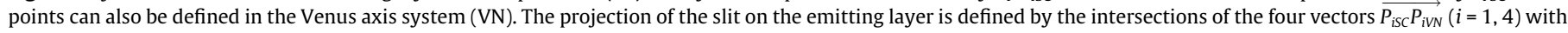
the sphere centered on Venus of radius $R_{\mathrm{VENUS}}+z_{\mathrm{PEAK}}$.

\subsection{Slit projection}

The area intercepted by a nadir observation performed by SPICAV is highly dependent on the position and orientation of the spacecraft with regard to Venus. We therefore use a two-axis system to define this surface, as presented in Fig. 1. Within the spacecraft axis system (SC in the following), a spatial bin is defined by four points: $\mathrm{P}_{1} \mathrm{SC}, \mathrm{P}_{2} \mathrm{SC}, \mathrm{P}_{3} \mathrm{SC}$ and $\mathrm{P}_{4} \mathrm{SC}$. Similarly, the focal point in this system of axis is defined as being $\mathrm{P}_{\mathrm{F}} \mathrm{SC}$. Four vectors may be defined from the focal point to each corner of the bin. In the SC axis system, we define them with $\mathrm{P}_{\mathrm{F}} \mathrm{SC}, \theta$ and $\phi$. We now call $\mathrm{VN}$ the system of axis defined by Venus. With the known geometry of the spacecraft in the VN system, one can transform $\mathrm{P}_{\mathrm{F}} \mathrm{SC}$ into $\mathrm{P}_{\mathrm{F}} \mathrm{VN}$. The focal point of SPICAV is now defined in the Venus axis system. The four vectors that link the focal point with each corner of the spatial bin are now defined in the VN system using the values of to $\mathrm{P}_{\mathrm{F}} \mathrm{VN}, \theta$ and $\phi$. We can then calculate the intersections of those four vectors with the sphere centered on Venus with a radius equal to $R_{\text {Venus }}+z_{\text {peak }}$, where $z_{\text {peak }}$ value is $115.5 \mathrm{~km}$, the average altitude of the NO emission peak (Stiepen et al., 2012). The four intersections of this sphere and the vectors that are closer to the spacecraft define a polygon in the emitting layer that is expressed in the VN system (i.e. in term of latitude and longitude).

\subsection{Viewing angle correction}

An ideal nadir observation is performed when the line of sight of SPICAV is strictly perpendicular to the emission layer and the planetary surface. However, during most observations, a non-zero angle exists between the line of sight and the normal, as presented in Fig. 2. The purpose of this correction is to calculate the equivalent vertical emission from a slant one, using an approximation to the Chapman function. The method is based on the expression described by Alexander et al. (1993) who approximated the Chapman function by:

$\operatorname{Ch}(R, H, \alpha)=\sec \left(\alpha-\frac{1}{y\left[1+(y / \sqrt{4 \pi})\left(\frac{\pi}{2}-\alpha\right)\right]}\right)$
With $\alpha$ the angle between the vertical direction and the line of sight, $H$ the scale height of the atmosphere and

$y=\sqrt{\frac{\pi\left(R+z_{\text {peak }}\right)}{2 H}}$

The equivalent vertical intensity $I_{V}$ is then given by

$I_{V}=I_{R E C} C h(R, H, \alpha)$

with $I_{R E C}$ the intensity of the emission observed at angle $\alpha$ from the vertical. A latitude/local time grid to populate is defined with a pixel size of $5^{\circ}$ latitude $\times 20 \mathrm{~min}$ LT. We first identify all the polygons that cover each pixel. We then calculate the surface ratio of each polygon covering the pixel. The intensity of each pixel is equal to the total intensity of all polygons covering that pixel, corrected for the chapman factor presented in the previous section and the surface ratio calculated for each polygon.

\subsection{Mapping procedure}

Since the observations used in this study were solely collected during the periods of time when the spacecraft was fully in the eclipse of Venus, gaps exist between groups of data. Observations performed when the spacecraft was illuminated show a diffuse scattered solar UV component ascribed to instrumental stray light and are therefore not used in this study. Fig. 3 illustrates the time distribution of the spectra included in our database. The number of spectra used in this study is indicated along the $y$ axis while the orbit number is indicated on the $x$ axis. One can easily isolate six groups of data. Groups one to six extend from orbit 1 to 500, 501 to 800,801 to 1000,1001 to 1300 and 1301 to 1600 and from 1601 to the last orbit number respectively.

Appendix A presents the noise subtraction method. It permits to estimate the noise level, as presented in Fig. 4. It is calculated as follows. The main noise components are $I_{d c}(i)$ and $I_{n u}(j)$, the spatial and temporal parts of the non-uniform dark current. One can express the error as

$E=\left\{1+\frac{I_{d c} \times I_{n u}}{100}+2\left[I_{c c} \times G\right]\right\}^{2}$ 


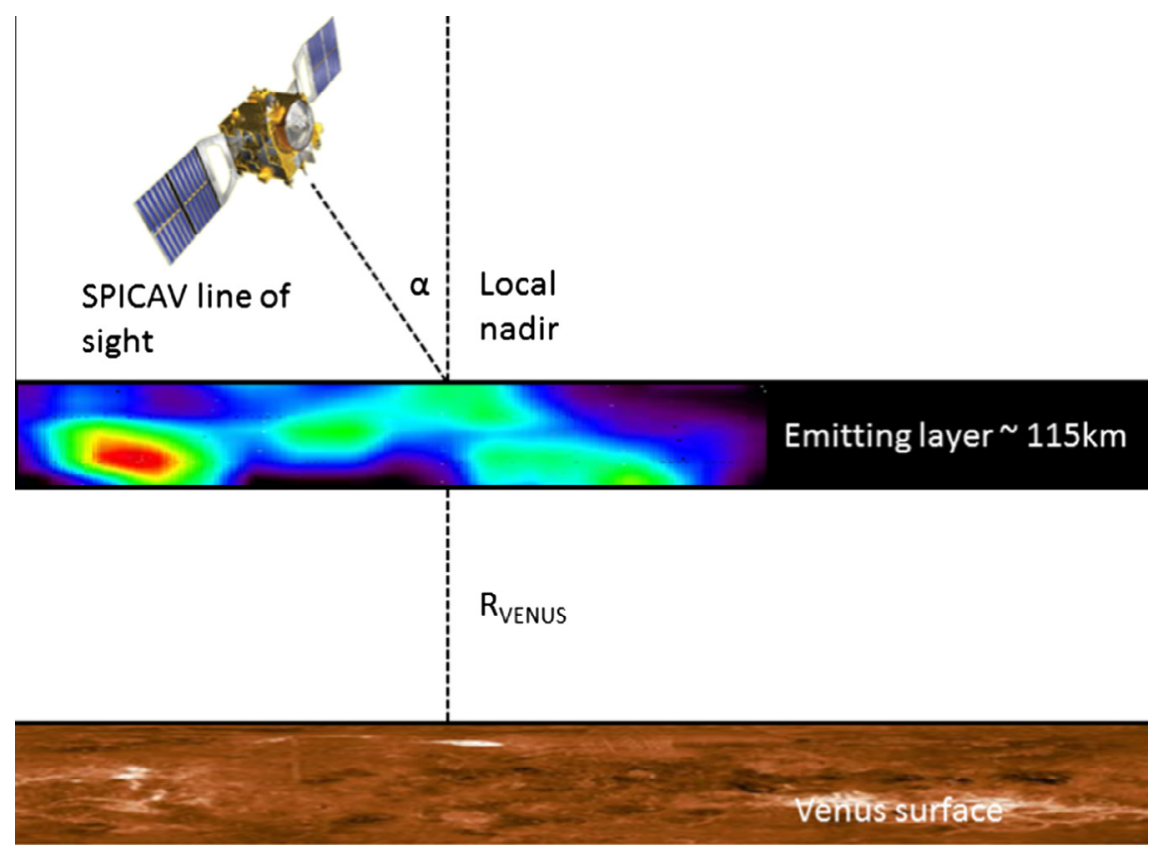

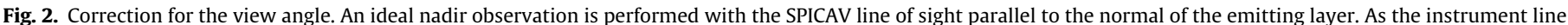
of sight may be tilted by an angle $\alpha$ with the normal of the emitting layer, a Chapman correction is applied to each spectrum to derive the nadir intensity.

where $E$ is the error, $I_{d c}$ and $I_{n u}$ explained in Appendix A, $I_{c c}$ is the cleaned and calibrated intensity (the black curve in Fig. 4) and $G$ is the gain during the observation. This value, $E$, is obtained for each spectrum.

The calculated noise level of each map pixel is then processed as an intensity value. The $1-\sigma$ noise level map in Fig. 5, panel $\mathrm{b}$ exhibits the uncertainty on the brightness map (panel a). The uncertainty does not exceed $1 \mathrm{kR}$. The signal-tonoise ratio in each pixel therefore ranges from $\sim 1$ up to more than 10 .

Fig. $5 \mathrm{a}$ is the global brightness map. It contains all usable observations that have been processed as explained before. All maps share the same pixel size of $5^{\circ}$ latitude $\times 20 \mathrm{~min}$ local time. A brightest region that extends from 01:00 to 03:30 local time and $25^{\circ} \mathrm{S}$ to $10^{\circ} \mathrm{N}$ appears in this nitric oxide emission map. Every

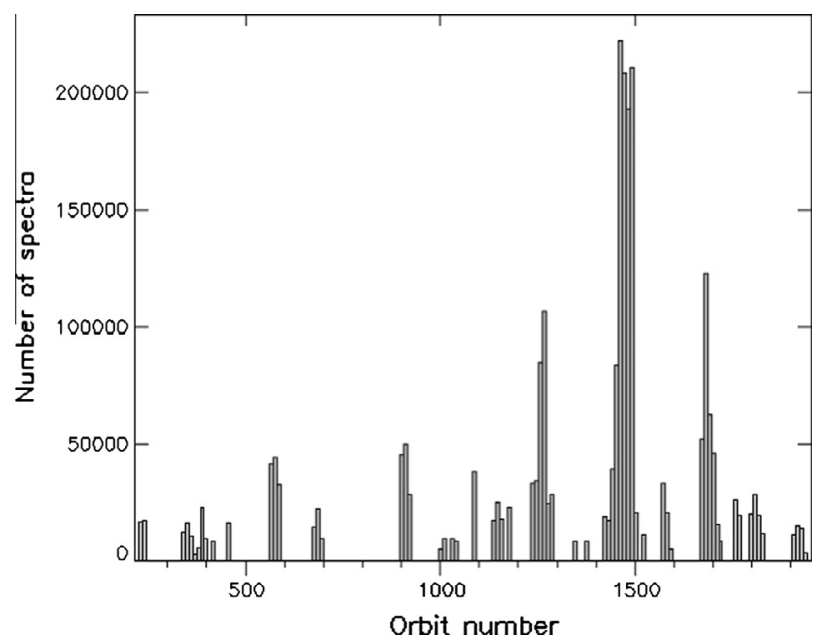

Fig. 3. Time distribution of the nadir NO nightglow spectra. Six groups of observations can be defined. Groups one to six extend from orbit 1 to 500,501 to 800,801 to 1000,1001 to 1300 and 1301 to 1600 and from 1601 to the last orbit number respectively. observations accumulated to produce this global map were added to produce the animation available as auxiliary material.

Panel $5 \mathrm{~b}$ is the mapping of the error associated with the intensity showed in Fig. 5a. It is calculated for each spectrum as presented in Appendix A and Fig. 4. The error value is then processed as described here above to produce the map.

The map of the statistics of the observations is presented in Fig. 5c. This figure shows the total observation time spent within each pixel. The non-uniform coverage of the observations causes the distinct inhomogeneity. Due to orbit characteristics, some regions have not been observed and are therefore left void of data. Pixels within the statistical brightest region were observed up to $\sim 2.5 \mathrm{~h}$ each, providing satisfactory statistics. In particular, the observation time spent in each pixel ranges from $\sim 300$ to $\sim 10,000 \mathrm{~s}$ in this region while the mean observation time per pixel is $\sim 1500 \mathrm{~s}$.

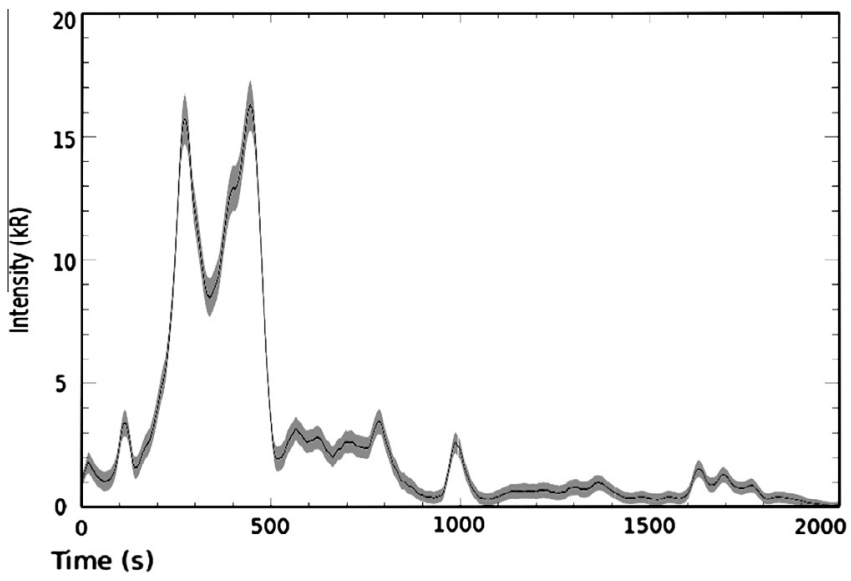

Fig. 4. Typical noise and intensity levels. Intensity variation of a nadir observation performed during orbit 387. The line of sight of SPICAV passes through a bright spot during the first $500 \mathrm{~s}$ of the observation. The cleaned and calibrated intensity distribution along the slit path is indicated by the black line while the light grey zone represents the $1-\sigma$ remaining noise level. 

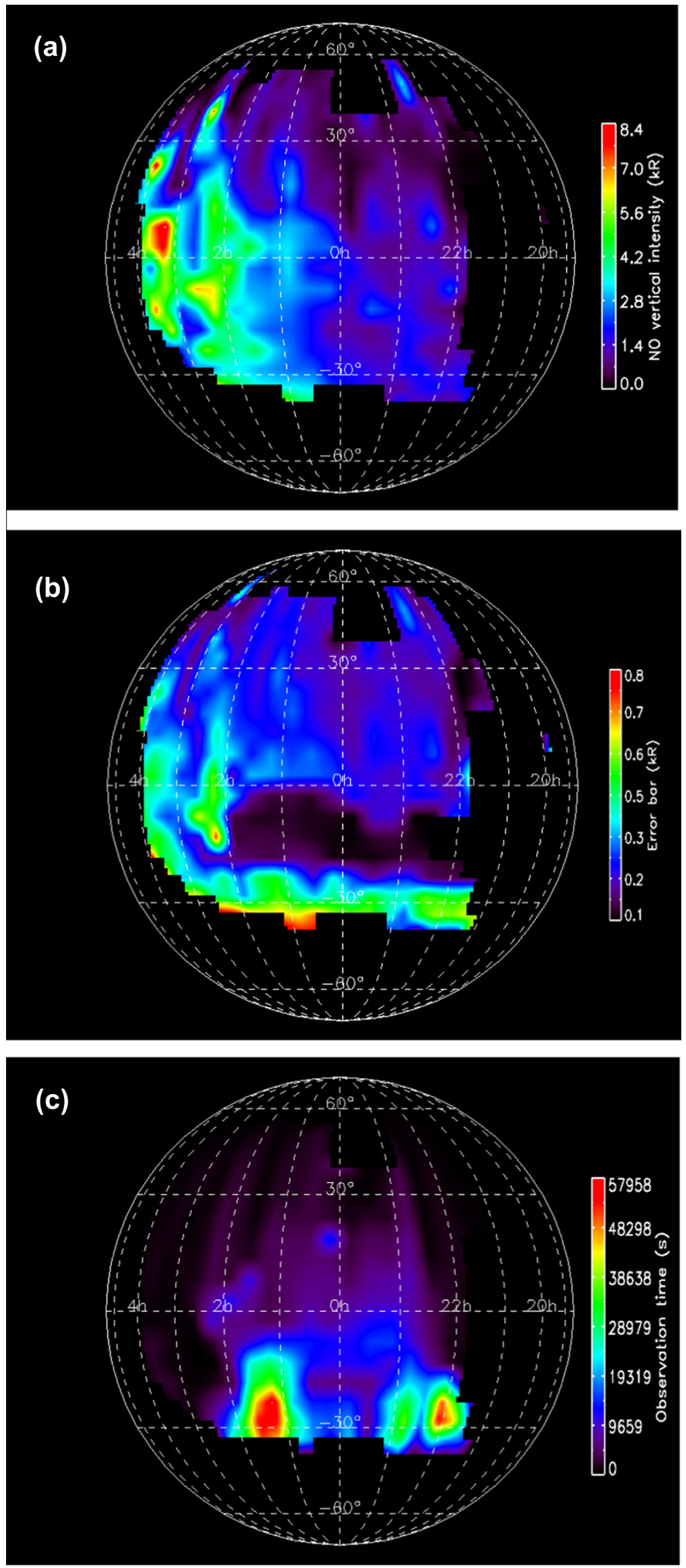

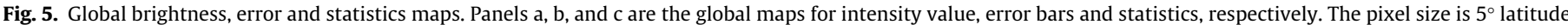
by $20 \mathrm{~min}$ of local time. 
Table 1

Intensities and $1-\sigma$ standard deviation for the NO nightglow emission from this study and comparison with values from PV-OUVS observations.

\begin{tabular}{|c|c|c|}
\hline Publications & Mean hemispheric intensity & Mean intensity in the brightest region \\
\hline This study & $1.9 \pm 1 \mathrm{kR}$ & $3.9 \pm 1.5 \mathrm{kR}$ (from $01: 00$ to $03: 30 \mathrm{LT}$ and from $25^{\circ} \mathrm{S}$ to $10^{\circ} \mathrm{N}$ ) \\
\hline Full NO emission & & $3.7 \pm 1.5 \mathrm{kR}$ (from $02: 15$ to $02: 45 \mathrm{LT}$ and from $\sim 20^{\circ} \mathrm{S}$ to $\sim 0^{\circ} \mathrm{N}$ ) \\
\hline $\begin{array}{l}\text { Bougher et al. (1990) } \\
\delta(0,1) \text { NO band }\end{array}$ & $0.45 \mathrm{kR}$ & $1.9 \mathrm{kR}$ (from $02: 15$ to $02: 45 \mathrm{LT}$ and from $\sim 20^{\circ} \mathrm{S}$ to $\sim 0^{\circ} \mathrm{N}$ ) \\
\hline $\begin{array}{l}\text { Bougher et al. (1990) } \\
\text { Full NO emission }\end{array}$ & $2 \pm 0.5 \mathrm{kR}$ & $8.6 \pm 2.7 \mathrm{kR}$ (from $02: 15$ to $02: 45 \mathrm{LT}$ and from $\sim 20^{\circ} \mathrm{S}$ to $\sim 0^{\circ} \mathrm{N}$ ) \\
\hline
\end{tabular}

\section{Discussion}

To compare with previous observations, we calculated the mean nightside hemispheric value of the NO emission and its lower bound. The mean value is obtained by averaging the intensity within all non-null pixels of the whole night hemisphere and dividing it by the number of pixels, while the lower bound is the mean value of the whole hemisphere with empty pixels set to zero. We obtain a mean value of $1.9 \mathrm{kR}$ and a lower bound of $0.51 \mathrm{kR}$ for the nightside hemisphere. The actual hemispheric mean emission is likely closer to the mean value obtained with the first method. The values for the brightest region (extending from 01:00 to 03:30 local time and $25^{\circ} \mathrm{S}$ to $10^{\circ} \mathrm{N}$ ) are respectively $3.9 \mathrm{kR}$ and $2.9 \mathrm{kR}$. They may be compared with those obtained by Stewart et al. (1980) who determined the mean intensity for the $\delta(0,1)$ band emission on the night hemisphere bounded by $60^{\circ} \mathrm{S}$ and $66^{\circ} \mathrm{N}$ and by $2000 \mathrm{LT}$ and $0400 \mathrm{LT}$ and within a bright region delimited by a box centered at $0230 \mathrm{LT}$ and slightly south of the equator. They used a box size of $9^{\circ}$ latitude $\times 1 \mathrm{~h}$ LT. The PV-OUVS values were subsequently recalculated (Bougher et al., 1990) and corrected. They found $0.48 \mathrm{kR}$ for the mean hemispheric nightside intensity of the $\delta(0,1)$ band and $1.9 \mathrm{kR}$ for the bright patch intensity of this emission band. The factor to convert the intensity of the $\delta(0,1)$ band to the total $\gamma$ and $\delta$ band system is derived from the measurements by Tennyson et al. (1986). They measured the NO nightglow spectrum during a rocket launch in 1981 and calculated the relative brightness of the various NO $\gamma$ and $\delta$ bands. The fraction emitted in the $\delta(0,1)$ band is $22 \%$ of the total NO spectrum.

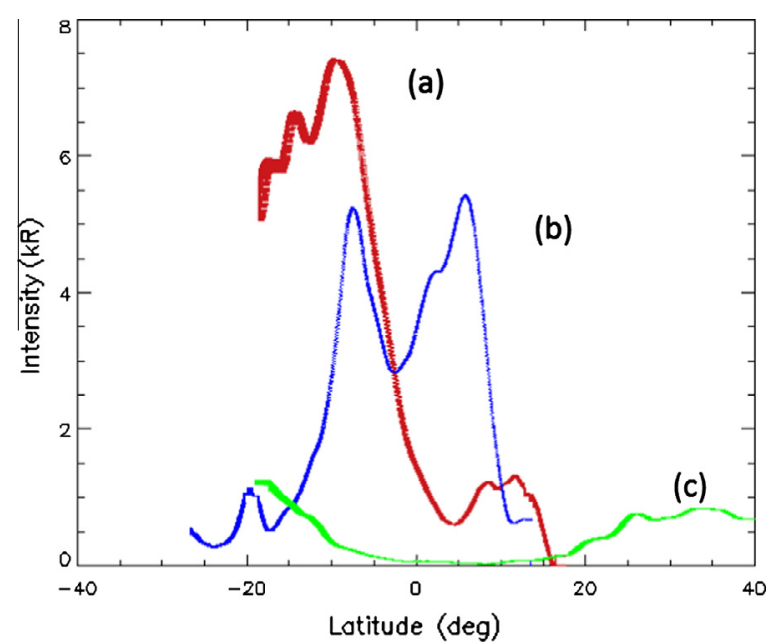

Fig. 6. Variability and latitudinal structure of the emission in the bright emission region. The curves show the intensity of the NO nadir emission for three different observations (orbits 393, 395 and 582) between 02:00 and 03:00 LT with similar solar conditions. While all three observations cross through the statistically bright region, different behaviors are observed. Curve (a) presents a secondary peak near $10^{\circ} \mathrm{N}$, curve (b) has two equally important peaks, while curve (c) has no maximum near $10^{\circ} \mathrm{S}$.
The total NO intensities derived from the work by Bougher et al. (1990) are therefore $2 \pm 0.5 \mathrm{kR}$ for the nightside hemisphere and $8.6 \pm 2.7 \mathrm{kR}$ for the brightest region. This implies a bright patch $\sim 4.5$ times brighter than the hemispheric mean brightness. The Venus Express observations yield a lower ratio of 2.1. The difference between the brightest region intensity values obtained in this study and those obtained by Bougher et al. (1990) for solar maximum conditions may be caused by the size and position of the box containing the brightest spot within which the values are calculated. We note however that these values are comparable and that the difference is probably not caused by a change in the solar activity, as the hemispheric values are similar. The data used in this study were accumulated during 2006 to 2011 and therefore cover low to medium solar conditions. Stewart and Barth (1979) did not demonstrate a clear correlation between the F10.7 index and the intensity values. We confirm that no correlation was found between the F10.7 index and the brightness of the NO emission.

Stewart and Barth (1979) and Bougher et al. (1990) defined a box centered on the bright spot situated at 02:30 LT and slightly south of the equator. The box size they used was $9^{\circ}$ latitude $\times 1 \mathrm{~h}$ LT. Similarly, the hemispheric mean value they obtained was calculated for emissions located within a box bounded by $60^{\circ} \mathrm{S}$ and $66^{\circ} \mathrm{N}$ and by 20:00 LT and 04:00 LT. We determined the average hemispheric values as explained here above (considering the whole hemisphere), while the brightest region in this study extends from 01:00 to 03:30 local time and $25^{\circ} \mathrm{S}$ to $10^{\circ} \mathrm{N}$. We recalculated from the SPICAV observations the mean values within the regions defined by Stewart and Barth (1979) and Bougher et al. (1990). The mean SPICAV value is unchanged at $1.9 \mathrm{kR}$, but the bright patch mean emission becomes $3.7 \mathrm{kR}$. We note that the average total NO intensities determined from the two missions are very close, within the error bars of the observations. The details of the comparison of these values are in Table 1.

A brighter region is clearly observed in the statistical brightness map: it is located between 01:00 and 03:30 local time and $25^{\circ} \mathrm{S}$ to $10^{\circ} \mathrm{N}$ latitude (see Fig. 5a). Data obtained close to the terminator have been removed as they may contain solar radiation contribution. As a consequence of the Venus Express orbit and operations, SPICAV nadir mode does not permit polar latitudes observations. A secondary bright region is visible in Fig. $5 \mathrm{a}$ around $40^{\circ} \mathrm{N}$ and 02:30 LT. Stewart et al. (1980) noted that bright patches are seen to occur randomly over a wide range of latitudes and local times. A secondary bright spot also appears on their Plate 4 at the same location as found from SPICAV data in this study. Stewart et al. (1980) explained the existence of the observed patchiness as the indication of spatial irregularities in the high-altitude circulation pattern. While the statistical brightest region is well defined, it appears that bright spots may occur at various locations on the nightside. Multiple spots have also been identified within the brightest region.

Fig. 6 shows examples of the variability of the airglow within the region of bright emission. The intensity of the NO emission is presented over a wide latitude range for three observations made from 02:00 to 03:00 LT during similar solar conditions. The three intensity curves clearly exhibit different behaviors in shape and 
intensity values. The middle curve (b) also exhibits multiple emission peaks. The variability and double peaks are larger than the error bars within the bright region, as the signal-to-noise ratio increases. This does not permit to perform a statistical study of the location of the double-peaked emissions. As well, a single observation covers a small area of the planet and a global image of multiple peaks and variability in the emission cannot be reproduced. However, Stewart and Barth (1979) already reported a dayto-day variability of the NO airglow emission using images taken $\sim 24 \mathrm{~h}$ apart. The $\mathrm{O}_{2}\left(\mathrm{a}^{1} \Delta \mathrm{g}\right)$ emission also shows a great deal of variability, as discussed by Soret et al. (2010). Clancy et al. (2012) reported Doppler wind measurements in the 95-115 km altitude range region from ${ }^{12} \mathrm{CO}$ and ${ }^{13} \mathrm{CO}$ at 346 and $330 \mathrm{GHz}$, respectively. These measurements show large temporal and spatial variations in the relative strength of the retrograde zonal and SSAS circulation close to $110 \mathrm{~km}$. They explained that these variations are very likely responsible for thermospheric temperature and composition local (both spatial and temporal) variability. The variability of the NO airglow emission is also apparent in the movie added as supplementary material. This animation presents the accumulation of data during the Venus Express mission. The bright spots are statistically located within the bright region, but their brightness and behavior (i.e. presence or absence of multiple peaks, brightest peak out of the bright region) is apparent during the movie.

Interesting features of the oxygen airglow described by Soret et al.'s (2010) Fig. 1 may be compared with the global NO airglow map presented in Fig. 5a. As was pointed out, the brightest region of $\mathrm{O}_{2}\left(\mathrm{a}^{1} \Delta\right)$ emission is centered on the subsolar point. The lack of spatial correlation between the $\mathrm{O}_{2}\left(\mathrm{a}^{1} \Delta\right)$ and the NO emissions observed in latitudinal cuts was discussed in Gérard et al. (2009). They used concurrent observations from SPICAV and VIRTIS instruments on board Venus Express to characterize the degree of correlation between those two emissions. They proposed that the absence of correlation is due to the presence of horizontal winds in the mesosphere-thermosphere transition region. For example, their Fig. 7 sketches the downward transport of atoms that recombine and generate airglow layers in different altitude regimes. The two-dimensional transport model by Collet et al. (2010) reproduced the separation between the NO and $\mathrm{O}_{2}\left(a^{1} \Delta\right)$ emissions following localized injection of a blob of $\mathrm{O}$ and $\mathrm{N}$ atoms. It is a consequence of the coupling between the horizontal and vertical transport processes. The long time scale of vertical transport and the different chemical lifetimes of the two species combine to produce a spatial separation of $\sim 2 \mathrm{~h}$ local time between the two emission brightest spots.

A comparison can also be made with the SPICAV limb observations of the nitric oxide emission. Stiepen et al. (2012) found a statistical drop of the peak VER with increasing latitude (from $\sim 1700 \mathrm{ph} \mathrm{cm}^{-3} \mathrm{~s}^{-1}$ down to $\sim 700 \mathrm{ph} \mathrm{cm}^{-3} \mathrm{~s}^{-1}$, corresponding to vertical intensities equal to $\sim 3.4 \mathrm{kR}$ and $\sim 1.4 \mathrm{kR}$ ) and a maximum at local times near 02:00 LT $\left(1250 \mathrm{ph} \mathrm{cm}^{-3} \mathrm{~s}^{-1}\right.$ at 02:00 LT to $435 \mathrm{ph} \mathrm{cm}^{-3} \mathrm{~s}^{-1}$ at 04:00 LT, corresponding to vertical intensities equal to $\sim 2.5 \mathrm{kR}$ and $\sim 0.9 \mathrm{kR})$. The mean VER profile showed in Stiepen et al. (2012) Fig. 4 have been vertically integrated. From this analysis, the mean corresponding vertical intensity is $\sim 1.9 \mathrm{kR}$. These results are in full agreement with the global map obtained in this work.

The results of this study raise the ongoing key question of the winds regime in the upper mesosphere-lower thermosphere region of the Venus nightside. The finding that the $\mathrm{O}_{2}$ airglow is not statistically shifted toward the morning terminator contradicts the assumption that the superrotation associated with the zonal circulation persists from the cloud top into the lower thermosphere. It apparently dies off at an altitude below the $\mathrm{O}_{2}$ airglow layer. Some other mechanism must be at play, which restores superrotation in the thermosphere and explains the NO airglow shift and other features such as the displaced He bulge. Niemann et al. (1980) proposed a flow field at thermospheric heights where the Coriolis force, coupled with the superrotation flow, diverts the flow vectors to create a stagnation point located around the equator and towards morning local times.

The need to explain the asymmetry in both thermospheric densities and NO nightglow was studied as soon as the early 1990s. In the VTGCM, Bougher et al. (1990) reproduced the observed superrotating winds by using a Rayleigh friction parameter. Alexander (1992) claimed that in situ forcing mechanism (i.e. gravity waves propagating from cloud-level to the thermosphere) causes the asymmetry in the global wind circulation. These waves could interact with the winds in the thermosphere region either by breaking or saturating, transferring momentum to the atmosphere. The recent work by Zalucha et al. (2013) shows that parameterizations of gravity waves in the VTGCM based on formalisms developed for the middle atmosphere cannot reproduce densities and nightglow emissions in agreement with the VEX observations. They concluded that gravity waves propagating from lower altitudes either break below $115 \mathrm{~km}$ or are reflected by the strong zonal wind existing in this altitude range. However, they argue that lower altitudes gravity waves may break in the $100-115 \mathrm{~km}$ altitude range and generate secondary waves able to transfer momentum to the atmosphere at $115 \mathrm{~km}$ or higher.

Another possibility to explain the winds regime in the upper mesosphere-lower thermosphere region of the Venus nightside is the coupling between the thermosphere and the plasma environment of Venus. Lundin et al. (2011) analyzed Aspera-4 Ion Mass Analyzer measurements to characterize the asymmetry of the solar wind and ionospheric ion flows near the planet. The main flow direction on the nightside is in the midnight-to-morning direction. They suggested that solar wind deposits momentum to the ionospheric plasma and provided clear suggestions of a downward transport of momentum. Below $\sim 400 \mathrm{~km}$ and down to $\sim 200 \mathrm{~km}$, the $\mathrm{O}^{+}$velocity decreases (while it was increasing down to $400 \mathrm{~km}$ ), indicating a momentum transfer between $\mathrm{O}^{+}$and the neutral atmosphere (mainly atomic oxygen). Below $\sim 160$ $140 \mathrm{~km}$, as $\mathrm{CO}_{2}$ becomes the main atmospheric constituent, momentum from $\mathrm{O}^{+}$ions may be transferred to the neutral atmosphere by charge exchange or ion drag. We raise the possibility that this transfer leads, via neutral-neutral collisions, to energy and momentum transfer that may reach lower regions of the atmosphere on a longer time basis and generate the zonal wind component causing the dawnward shift of the statistical NO bright spot.

\section{Conclusions}

We have obtained the first map of the ultraviolet NO Venus nightglow since the Pioneer-Venus era and the only one for low solar activity (from 2006 to end 2010) and low to medium solar activity (during the year 2011). It is based on data collected in the nadir direction with the SPICAV instrument. The Venus NO nightglow exhibits a region with enhanced emission intensity extending between 01:00 and 03:30 local time and $25^{\circ} \mathrm{S}$ to $10^{\circ}$ $\mathrm{N}$ latitude. Intensities as high as $\sim 20 \mathrm{kR}$ have been observed in individual observations. The mean vertical intensity of this statistically bright region is found to be $3.9 \mathrm{kR}$, while the hemispheric emission rate of the NO emission is $1.9 \mathrm{kR}$. Single observations have shown the existence of multiple emission peaks along latitudinal cuts within the bright region as well as the possibility for the brightest spot to move in a limited time within this region.

The comparison with the $\mathrm{O}_{2}\left(\mathrm{a}^{1} \Delta\right)$ emission distribution and the He bulge raises the question of the mechanisms that produces the superrotation of Venus' upper atmosphere. This superrotation present at cloud top level $(\sim 70 \mathrm{~km})$ disappears at the altitude of 
the $\mathrm{O}_{2}\left(\mathrm{a}^{1} \Delta\right)$ emission $(\sim 95 \mathrm{~km})$. The NO map presented in this study is in agreement with the results from Stewart and Barth (1979) based on PV-OUVS observations and confirms the existence of a statistical superrotation in the thermosphere at $\sim 115 \mathrm{~km}$. It is also in agreement with the presence of the helium bulge (from $\sim 150 \mathrm{~km}$ to $\sim 250 \mathrm{~km}$ ) which indicates the existence of an atmospheric superrotation at higher altitudes. Two possible mechanisms of momentum transfer may explain superrotation near $115 \mathrm{~km}$. Recent results from VTGCM simulations (Zalucha et al., 2013) show that gravity waves emerging from lower altitudes break in the $100-115 \mathrm{~km}$ altitude range, transferring momentum and energy to the atmosphere in this region. On the other hand, momentum transfer from ionospheric flow may propagate from higher altitudes, as proposed by Lundin et al. (2011) on the basis of recent ASPERA-4 plasma measurements. These two mechanisms may coexist to produce superrotation at the altitude of the NO emission, while none being able to drive the atmosphere to superrotate below $100 \mathrm{~km}$. Further modeling is needed to assess the validity of this mechanism and its efficiency as a function of altitude.

\section{Acknowledgments}

The authors thank the SPICAV and the Venus Express teams for the excellent quality of their work. This research was supported by the PRODEX program of the European Space Agency (ESA) managed with the help of the Belgian Space Policy Office (BELSPO). The construction of the SPICAV instrument was funded by CNRS, CNES and ESA/PRODEX-BELSPO. We are also grateful to Aurélie Reberac and Benoit Heylens for their useful help in data management.

\section{Appendix A. Noise subtraction}

A signal recorded by SPICAV can be decomposed into the sum of several contributions. Several of these contributions must be evaluated and subtracted from the recorded signal to provide the signal emitted by the source. The recorded signal can be written

$I_{\text {rec }}(i, j)=I_{\text {em }}(i, j)+I_{\text {dcnu }}(i, j)+I_{\text {off }}(j)+I_{\text {read }}(i, j)+I_{\text {par }}(i, j)$

where $I_{r e c}$ is the signal recorded by SPICAV, $I_{e m}$ is the emitted signal to be retrieved, $I_{d c n u}$ is the signal due to non-uniform dark current, $I_{\text {off }}$ is the offset, $I_{\text {read }}$ is the reading noise, $I_{\text {par }}$ is an eventual stray signal and $i$ and $j$ are the temporal and pixel index, respectively. The non-uniform dark current is proportional to the temperature of the CCD. It is created by the formation of electron-hole pairs in the semi-conductor of the CCD. Its intensity varies from a pixel to another and with time. It can be written as

$I_{d c n u}(i, j)=I_{d c}(i) \times I_{n u}(j)$

with $I_{d c}$ the mean dark current value at time $i$ and $I_{n u}$ the pattern of the variation of the dark current with the wavelength.

In order to evaluate $I_{d c n u}(i, j)$, one uses technological observations. They are performed with a null amplification of the UV image intensifier so that no external signal is recorded. Moreover, if no stray signal is present, the recorded signal may be expressed as

$I_{\text {rec }}(i, j)=I_{d c}(j) \times I_{n u}(j)+I_{\text {off }}(j)+I_{\text {read }}(i, j)$

The masked pixels record a signal equal to the sum of the offset signal plus an estimate of the mean dark current signal $\left(\operatorname{est}\left(I_{d c n u}(i, j)\right)\right.$. A time-dependent linear relationship exists between the mean signal recorded by the masked pixels and the sum of the offset and dark current signals

$I_{d c}(i) \times I_{n u}(j)+I_{o f f}(j)=a(j) \times I_{p x m}(i)+b(j)$ where $I_{p x m}(i)$ is the mean signal recorded by the masked pixels. Eq. (12) is approximated using a least-squares fit method to retrieve $a$ and $b$ at each wavelength. Since $\operatorname{est}\left(I_{d c n u}(i, j)\right)$ is equal to zero when $\left.I_{d c n u}(i, j)\right)$ is null, Eq. (12) leads to

$I_{d c}(i) \times I_{n u}(j)+I_{o f f}(j)=a(j) \times\left[\operatorname{est}\left(I_{d c n u}(i, j)+I_{o f f}(j)\right]+b(j)\right.$

$0+I_{o f f}(j)=a(j) \times\left[0+I_{o f f}(j)\right]+b(j)$

$I_{o f f}(j)=\frac{b(j)}{1-a(j)}$

$I_{d c n u}(i, j)$ and $I_{o f f}(j)$ are calculated separately using Eqs. (12) and (15). One can now retrieve the sum of the emitted signal plus the signal due to reading noise from the recorded signal

$I_{\text {em }}(i, j)+I_{\text {read }}(i, j)=I_{\text {rec }}(i, j)-\left[a(j) \times I_{p x m}(i)+b(j)\right]$

In order to retrieve the emitted signal, one must now estimate the signal due to reading noise. An excellent approximation is given by

$I_{\text {read }}(i, j)=A \times \sin (\omega(j)+\varphi(i))$

At each time $(i)$, we approximate the recorded signal outside of the NO waveband (i.e. where $I_{e m}(i, j)=0$ ) by this expression using a least-squares fit. Parameters $\omega$ and $\varphi$ are then used to subtract the reading noise from the whole spectrum at time $i$.

The remaining noise that may contaminate a recorded signal is the stray signal, $I_{\text {par }}$. We eliminate the occurrence of a solar direct and indirect contamination by using observations performed when the spacecraft is within the shadow cone of Venus (i.e. when SPICAV is in the eclipse). Nevertheless, the most frequent source of stray signals is the impacts of gamma rays on the CCD. The pattern of a gamma ray signal is a SPICAV spectrum is a very high intensity in a specific pixel or small group of pixels within a short period of time. The affected pixels can be easily determined by a visual examination. They are replaced by the mean intensity value recorded by the adjacent pixels.

\section{Appendix B. Supplementary material}

Supplementary data associated with this article can be found, in the online version, at http://dx.doi.org/10.1016/j.icarus.2013. 05.031.

\section{References}

Alexander, M.J., 1992. A mechanism for the Venus thermospheric superrotation. Geophys. Res. Lett. 19, 2207-2210. http://dx.doi.org/10.1029/92GL02110.

Alexander, M., Stewart, A.I.F., Solomon, D.S.C., Bougher, S.W., 1993. Local time asymmetries in the Venus thermosphere. J. Geophys. Res. 98, 10849-10871.

Bertaux, J.L. et al., 2007. SPICAV on Venus Express: Three spectrometers to study the global structure and composition of the Venus atmosphere. Planet. Space Sci. $55,1673-1700$.

Bougher, S.W., Gérard, J.C., Stewart, A.I.F., Fesen, C.G., 1990. The Venus nitric oxide night airglow - Model calculations based on the Venus Thermospheric General Circulation Model. J. Geophys. Res. 95, 6271-6284.

Bougher, S.W., Alexander, M.J., Mayr, H.G., 1997. Upper atmosphere dynamics: Global circulation and gravity waves. In: Bougher, S.W., Hunten, D.M., Phillips, R.J. (Eds.), Venus II: Geology, Geophysics, Atmosphere, and Solar Wind, Environment, p. 259

Bougher, S.W., Rafkin, S., Drossart, P., 2006. Dynamics of the Venus upper atmosphere: Outstanding problems and new constraints expected from Venus Express. Planet. Space Sci. 54, 1371-1380. http://dx.doi.org/10.1016/ j.pss.2006.04.023.

Brecht, A.S., Bougher, S.W., Gérard, J.C., Parkinson, C.D., Rafkin, S., Foster, B., 2011. Understanding the variability of nightside temperatures, NO UV and $\mathrm{O}_{2}$ IR nightglow emissions in the Venus upper atmosphere. J. Geophys. Res. 116, 8004.

Clancy, R.T., Sandor, B.J., Moriarty-Schieven, G., 2012. Circulation of the Venus upper mesosphere/thermosphere: Doppler wind measurements from 2001-2009 inferior conjunction, sub-milmlimeter $\mathrm{CO}$ absorption line observations. Icarus $217,794-812$

Collet, A., Cox, C., Gérard, J.-C., 2010. Two-dimensional time-dependent model of the transport of minor species in the Venus night side upper atmosphere. Planetary and Space Science 58, 1857-1867. 
436

A. Stiepen et al./Icarus 226 (2013) 428-436

Crisp, D., Meadows, V. S., Bézard, B., de Bergh, C., Maillard, J.-P., Mills, F. P., 1996. Ground-based near-infrared observations of the Venus nightside: $1.27-\mu \mathrm{m}$ O2(a $1 \Delta \mathrm{g}$ ) airglow from the upper atmosphere. J. Geophys. Res., 101, E2, 4577-4593.

Dickinson, R.E., Ridley, E.C., 1977. Venus mesosphere and thermosphere temperature structure. Day-night variations. Icarus 30, 163-178. http:// dx.doi.org/10.1016/0019-1035(77)90130-0.

Drossart, P. et al., 2007. Scientific goals for the observation of Venus by VIRTIS on ESA/Venus Express mission. Planet. Space Sci. 55, 1653-1672.

Feldman, P.D., Moos, H.W., Clarke, J.T., Lane, A.L., 1979. Identification of the UV nightglow from Venus. Nature 279, 221-222.

Gerard, J.C., Stewart, A.I.F., Bougher, S.W., 1981. The altitude distribution of the Venus ultraviolet nightglow and implications on vertical transport. Geophys. Res. Lett. 8, 633-636.

Gérard, J.C., Cox, C., Saglam, A., Bertaux, J.L., Villard, E., Nehmé, C., 2008. Limb observations of the ultraviolet nitric oxide nightglow with SPICAV on board Venus Express. J. Geophys. Res. 113, E00B03.

Gérard, J.C. et al., 2009. Concurrent observations of the ultraviolet nitric oxide and infrared $\mathrm{O}_{2}$ nightglow emissions with Venus Express. J.Geophys. Res. 114, E00B44

Lellouch, E., Clancy, T., Crisp, D., Kliore, A.J., Titov, D., Bougher, S.W., 1997. Monitoring of mesospheric structure and dynamics. In: Bougher, S.W., Hunten, D.M., Phillips, R.J. (Eds.), Venus II: Geology, Geophysics, Atmosphere, and Solar Wind, Environment, p. 295.

Lundin, R., Barabash, S., Futaana, Y., Sauvaud, J.-A., Fedorov, A., Perez-de-Tejada, H., 2011. Ion flow and momentum transfer in the Venus plasma environment. Icarus 215 (2), 751-758.

Niemann, H.B., Kasprzak, W.T., Hedin, A.E., Hunten, D.M., Spencer, N.W., 1980. Mass spectrometric measurements of the neutral gas composition of the thermosphere and exosphere of Venus. J. Geophys. Res. 85, 7817-7827.

Ohtsuki, S., Iwagami, S., Sagawa, H., Kasaba, Y., Ueno, M., Imamura, T., 2005. Ground-based observation of the Venus 1.27- $\mu \mathrm{m} \mathrm{O}_{2}$ airglow. Advances in Space Research 36,2038-2042. http://dx.doi.org/10.1016/j.asr.2005.05.078. -.

Ohtsuki, S., Iwagami, S., Sagawa, H., Ueno, M., Kasaba, Y., Imamura, T., Yanagisawa, K., Nishihara, E., 2008. Distributions of the Venus $1.27-\mu \mathrm{m} \mathrm{O}_{2}$ airglow and rotational temperature. Plan. Space Sci. 56, 1391-1398.
Piccioni, G., Zasova, L., Migliorini, A., Drossart, P., Shakun, A., Munoz, A.G., Mills, F.P., Cardesin-Moinelo, A., 2009. Near-IR oxygen nightglow observed by VIRTIS in the upper Venus atmosphere. J. Geophys. Res. 114, E00B38. http://dx.doi.org/ 10.1029/2008JE003133.

Royer, E., Montmessin, F., Bertaux, J.L., 2010. NO emissions as observed by SPICAV during stellar occultations. Planet. Space Sci. 58, 1314-1326.

Schubert, G. et al., 1980. Structure and circulation of the Venus atmosphere. J. Geophys. Res. 85, 8007-8025. http://dx.doi.org/10.1029/JA085iA13p08007.

Schubert, G. et al., 2007. Venus atmosphere dynamics: A continuing enigma. In: Esposito, L.W., Stofan, E.R., Cravens, T.E. (Eds.), Exploring Venus as Terrestrial Planet, Geophys. Monogr. Ser., vol. 176. AGU, Washington, DC, pp. 121-138.

Soret, L., Gérard, J.C., Piccioni, G., Drossart, P., 2010. Venus OH nightglow distribution based on VIRTIS limb observations from Venus Express. Geophys. Res. Lett. 370, L06805.

Stewart, A.I., Barth, C.A., 1979. Ultraviolet night airglow of Venus. Science 205, 5962.

Stewart, A.I.F., Gérard, J.C., Rusch, D.W., Bougher, S.W., 1980. Morphology of the Venus ultraviolet night airglow. J. Geophys. Res. 85, 7861-7870.

Stiepen, A., Soret, L., Gérard, J.-C., Cox, C., Bertaux, J.-L., 2012. The vertical distribution of the Venus NO nightglow: Limb profiles inversion and onedimensional modeling. Icarus 220, 981-989.

Svedhem, H. et al., 2007. Venus Express: The first European mission to Venus. Icarus $55,1636-1652$.

Tennyson, P.D., Feldman, P.D., Hartig, G.F., Henry, R.C., 1986. Near-midnight observations of nitric oxide $\delta$ - and $\gamma$-band cheluminescence. J. Geophys. Res. 91 (A9), 10,141-10,146.

Titov, D.V. et al., 2006. Venus Express science planning. Planet. Space Sci. 54, 12791297.

Zalucha, A.M., Brecht, A.S., Rafkin, S., Bougher, S.W., Alexander, M.J., 2013. Incorporation of a Gravity Wave Momentum Deposition Parameterization into the VTGCM. J. Geophys. Res. 118. http://dx.doi.org/10.1029/2012JE004168. 